\title{
USO DE CENIZA DE BAGAZO DE CAÑA COMO ADICIÓN EN MEZCLAS DE CONCRETO ELABORADAS CON MATERIAL RECICLADO: EVALUACIÓN ELECTROQUÍMICA
}

\author{
C. BERNABÉ ${ }^{1}$, A. ZALAPA ${ }^{1}$, E. M. ALONSO ${ }^{2}$, V. H. BLANCAS ${ }^{2}$ \\ ${ }^{1}$ Doctorado en Ciencias en Ingeniería Química, Facultad de Ingeniería Química, \\ Universidad Michoacana de San Nicolás de Hidalgo, Morelia, México. \\ ${ }^{2}$ Laboratorio de Materiales, Facultad de Ingeniería Civil, \\ Universidad Michoacana de San Nicolás de Hidalgo, Morelia, México.
}

\begin{abstract}
RESUMEN
Ante la imperante necesidad de contribuir en el cuidado del medio ambiente es necesario un cambio de paradigma en el uso de materiales. Para este trabajo se elaboraron especímenes de concreto de $5 \mathrm{~cm} \times 10 \mathrm{~cm} \times 20 \mathrm{~cm}$, con barras de acero de 3/8" de diámetro. Se utilizó ceniza de bagazo de caña $(\mathrm{CBC})$ como sustituto parcial del cemento y concreto triturado obtenido de demoliciones, con relaciones agua/cemento de 0.45 y 0.65 . Se midieron potenciales de corrosión y velocidad de corrosión en los ciclos húmedo-seco, utilizando solución de $\mathrm{NaCl}$ al 3.5\%. Los valores más altos de velocidad de corrosión los presentó el concreto con relación a/c de 0.65 . El material reciclado tiene alta porosidad, sin embargo, se puede emplear en estructuras no expuestas a medios agresivos.
\end{abstract}

Palabras clave: concreto reforzado; material reciclado; sustitución; durabilidad; velocidad de corrosión.

\begin{abstract}
Given the prevailing need to contribute to the care of the environment, a paradigm shift in the use of materials is necessary. Specimens of $5 \mathrm{~cm} x 10 \mathrm{~cm} x 20 \mathrm{~cm}$, with $3 / 8$ "diameter steel bar concrete were developed for this work. Bagasse cane ash (CBC) was used as a partial substitute for cement and crushed concrete obtained from demolitions, with water / cement ratios of 0.45 and 0.65 . Corrosion potentials and corrosion rate were measured in wet-dry cycles, using $3.5 \% \mathrm{NaCl}$ solution. The highest values of corrosion rate were presented by the concrete with a / c ratio of 0.65 . The recycled material has high porosity, however it can be used in structures not exposed to aggressive media.
\end{abstract}

Keywords: reinforced concrete; recycled material; substitution; durability; corrosion rate.

\section{RESUMO}

Dada a necessidade prevalecente de contribuir para o cuidado do meio ambiente, é necessária uma mudança de paradigma no uso de materiais. Para este trabalho foram confeccionados espécimes de concreto de $5 \mathrm{~cm} \times 10 \mathrm{~cm} \times 20 \mathrm{~cm}$, com barras de aço de $3 / 8$ "de diâmetro. A cinza de cana-de-bagaço (CBC) foi utilizada como substituto parcial do cimento e do concreto triturado obtido a partir de demolições, com razões água / cimento de 0,45 e 0,65. Os potenciais de corrosão e a taxa de corrosão foram medidos em ciclos úmidos e secos, usando solução de $\mathrm{NaCl}$ a 3,5\%. Os maiores valores de velocidade de corrosão foram apresentados pelo concreto com uma relação a / c de 0,65. O material reciclado possui alta porosidade, porém pode ser usado em estruturas não expostas a meios agressivos. Palavras-chave: concreto armado; material reciclado; substituição; durabilidade; taxa de corrosão. 


\section{INTRODUCCIÓN}

El concreto es uno de los materiales de construcción más utilizados en todo el mundo, gracias a sus cualidades de resistencia a la compresión y durabilidad, se obtiene de la mezcla de agua, cemento, arena y grava. Las obras de construcción civil, aun siendo apropiadas para las cargas y exigencias para las cuales fueron proyectadas, no dejan de presentar algunos problemas serios de mantenimiento, durante su utilización (DURAR, 1998). El concreto puede soportar de manera satisfactoria los esfuerzos a compresión, pero su capacidad de resistir esfuerzos a tensión es menor, aproximadamente de $8 \%$ a $12 \%$ de la resistencia a la compresión (Kosmatka, Kerkhoff, Panarese, \& y Tanesi, 2004). Es esta necesidad de poder resistir dichos esfuerzos a tensión lo que ha llevado a la combinación de concreto con acero de refuerzo, logrando un mayor desempeño de estos materiales en la construcción. Se considera que entre un $25 \%$ y un $30 \%$ de la producción mundial de hierro es destruido anualmente por la corrosión. Las estimaciones avanzan aún más y los costos asociados al fenómeno de corrosión oscilan cerca del 4\% del PIB de los países más industrializados (Pancorbo, 2013). El uso de materiales suplementarios tiene relevancia ambiental, lo que repercute en una disminución de la cantidad de cemento que se utiliza. La producción de una unidad de cemento genera aproximadamente una unidad de $\mathrm{CO}_{2}$ que es emitida a la atmosfera, lo que repercute en el efecto invernadero (Escalante \& Navarro Gómez, 2011; Ruchansky, 2013), y globalmente la industria cementera genera el 7\% de las emisiones de $\mathrm{CO}_{2}$ según el Intergovernmental Panel on Climate Change del 2007.

\section{MATERIALES}

\subsection{Material Cementicio}

El cemento utilizado fue CPC 30R RS (ASTM-C150, 2005). Como material complementario o sustituto parcial del cemento se utilizó la CBC. En la Tabla 1 se muestran los resultados de fluorescencia de rayos X del CPC y la CBC.

Tabla 1. Composición química en porcentaje, en masa, de los materiales utilizados en las sustituciones, obtenidas por Fluorescencia de rayos X.

\begin{tabular}{|l|c|c|c|c|c|c|c|c|c|c|c|}
\hline Muestra & $\mathrm{SiO}_{2}$ & $\mathrm{Al}_{2} \mathrm{O}_{3}$ & $\mathrm{TiO}_{2}$ & $\mathrm{Fe}_{2} \mathrm{O}_{3}$ & $\mathbf{M g O}$ & $\mathrm{MnO}$ & $\mathbf{C a O}$ & $\mathbf{N a}_{2} \mathrm{O}$ & $\mathrm{K}_{2} \mathrm{O}$ & $\mathbf{S O}_{3}$ & PXC/PPI \\
\hline CBC & 56.62 & 4.293 & 0.422 & 5.488 & 1.573 & 0.227 & 2.013 & 0.24 & 2.178 & 0.244 & 26.65 \\
\hline $\begin{array}{l}\text { CPC } \\
\text { 30R RS }\end{array}$ & 23.88 & 4.997 & 0.217 & 2.972 & 0.88 & 0.096 & 54.6 & 1.23 & 1.666 & 3.791 & 5.54 \\
\hline
\end{tabular}

La CBC se utilizó previo triturado y tamizado, usando las partículas que pasan la malla ASTM No. 200. En la Figura 1 se muestra la distribución granulométrica de la CBC comparada con la de CPC 30R. Se puede observar que la CBC presenta tamaños de partícula mayores que el CPC, lo que infiere velocidad de reacción menor, debido a la disminución del área superficial y la dificultad para lograr la nucleación de los hidratos en las partículas de sílice. El uso de la CBC tiene como desventaja el uso de energía para disminuir el tamaño de la partícula y lograr mayor reactividad (Jagadesh \& et. al., 2018). La norma ASTM C618 clasifica a la CBC como una puzolana N (ASTM-C618, 2003). 
Distribución Granulométrica de las partículas de CBC Y CPC.

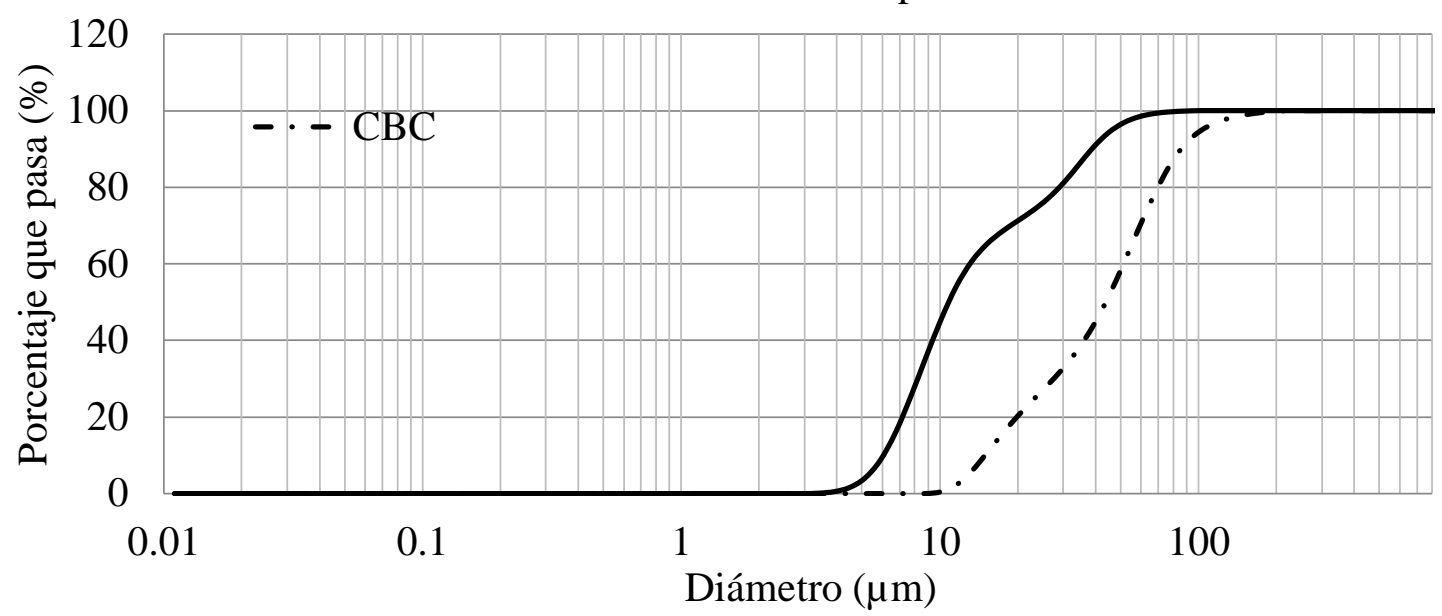

Figura 1 Distribución granulométrica de las partículas de CBC Y CPC empleadas en la elaboración del concreto.

Se realizaron análisis morfológicos y elementales por medio de microscopia electrónica de barrido (MEB). En la Figura 2, izquierda, se observan las formas alargadas y porosas de la CBC, lo cual confirma que después de la calcinación las partículas conservan la forma de la fibra de la caña. En el análisis EDS se observa el silicio como elemento predominante seguido del oxígeno.

$\mathrm{La} \mathrm{CBC}$ en estudio presenta un carácter ácido intermedio $\left(55 \%<\mathrm{SiO}_{2}<65 \%\right)$, lo cual puede resultar en una reducción de la alcalinidad de la mezcla de concreto (Valdez Tamez, Das Roy, \& Rivera Villareal, 2004).
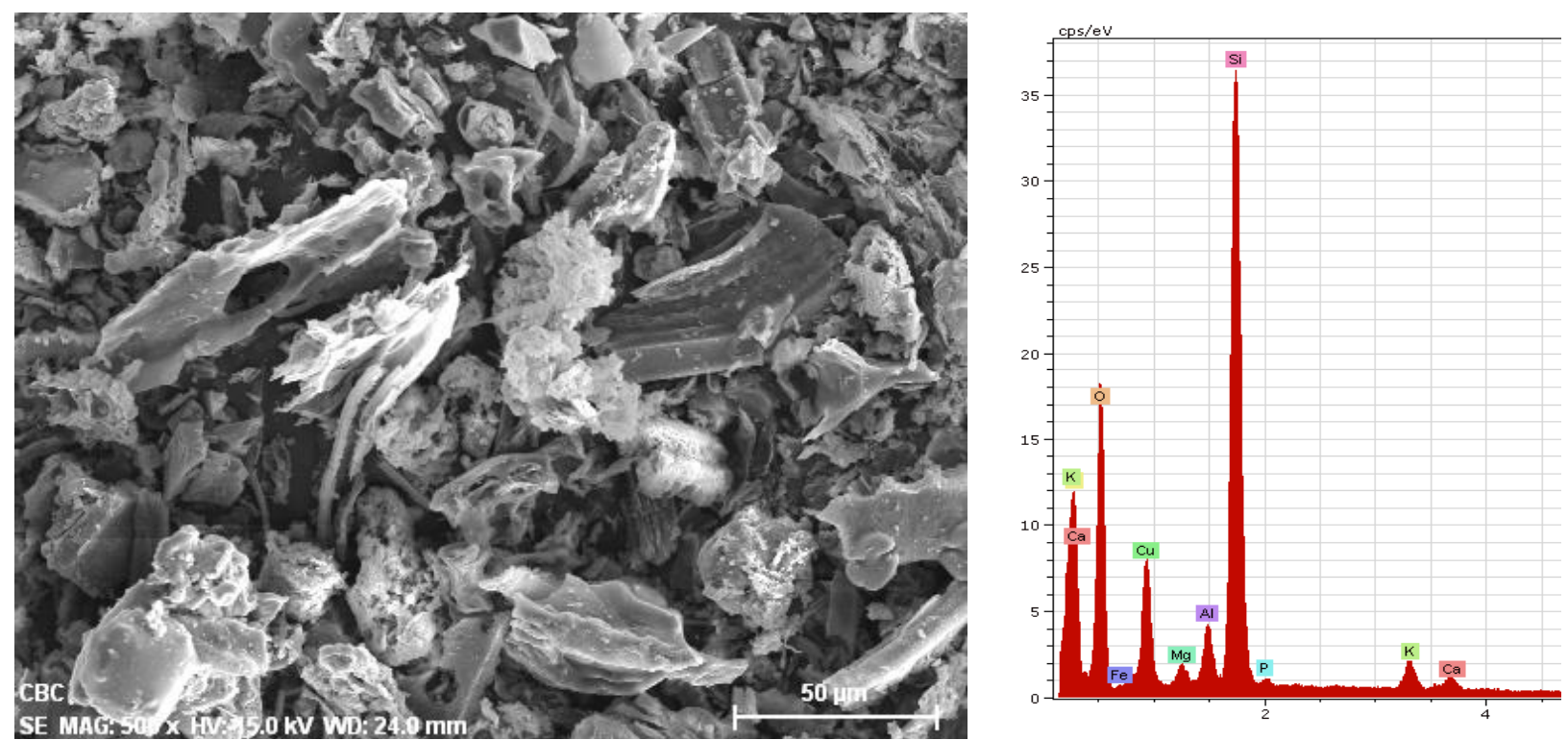

Figura 2 Izquierda, morfología de la CBC analizada en el MEB a 500x; derecha, análisis EDS.

\subsection{Material Inerte}

\subsubsection{Agregado grueso}

Se utilizaron como agregado grueso grava natural triturada y concreto reciclado. Las características de estos materiales se muestran en la Tabla 2. 
Tabla 2. Propiedades físicas de la grava natural triturada y del material reciclado

\begin{tabular}{|c|c|c|}
\hline Propiedades Físicas & Grava Natural Triturada (GNT) & Concreto reciclado (GCR) \\
\hline M.V.S.S (kg/m3) & 1396 & 1148 \\
\hline M.V.S.V (kg/m3) & 1549 & 1280 \\
\hline Densidad (g/cm3) & 2.64 & 2.16 \\
\hline Absorción (\%) & 1.6 & 6 \\
\hline T.M (pulgadas) & $1 / 2$ & $3 / 4$ \\
\hline
\end{tabular}

El material GCR presenta propiedades físicas menos deseables que el material GNT. Se observa una alta porosidad en el reciclado, por lo que su empleo no resulta viable en concretos expuestos a ambientes agresivos, sobre todo en zonas costeras.

\subsubsection{Agregado Fino}

El agregado fino (arena) es volcánica, se obtuvo del banco de material La Unión, los resultados de la caracterización se presentan en la Tabla 3. Esta arena se utilizará para todas las mezclas.

Tabla 3. Propiedades físicas de la arena

\begin{tabular}{|lr|}
\hline \multicolumn{2}{|c|}{ Arena Banco "La unión" - Joyitas } \\
\hline M.V.S.S $\left(\mathrm{kg} / \mathrm{m}^{3}\right)$ & 1343 \\
\hline M.V.S.V $\left(\mathrm{kg} / \mathrm{m}^{3}\right)$ & 1496 \\
\hline Densidad $\left(\mathrm{g} / \mathrm{cm}^{3}\right)$ & 2.4 \\
\hline Absorción $(\%)$ & 3.5 \\
\hline M. F. (pulgadas) & 2.71 \\
\hline
\end{tabular}

\subsection{Proporciones de las mezclas}

En la Tabla 4 se listan las proporciones de los materiales para la mezcla base usando el método de proporcionamiento del American Concrete Institute (ACI) para un metro cúbico de concreto.

Tabla 4. Proporciones de los materiales empleados para la elaboración de un metro cúbico de concreto. Dosificación para el material natural y reciclado con relaciones a/c de 0.45 y 0.65 .

\begin{tabular}{|lll|ll|}
\hline \multicolumn{3}{l}{ Grava Reciclada $($ GCR) } & \multicolumn{2}{c|}{ Grava Triturada $($ GNT) } \\
\hline Material & $\mathbf{a} / \mathbf{c}=\mathbf{0 . 4 5}$ & $\mathbf{a} / \mathbf{c}=\mathbf{0 . 6 5}$ & $\mathbf{a} / \mathbf{c}=\mathbf{0 . 4 5}$ & $\mathbf{a} / \mathbf{c}=\mathbf{0 . 6 5}$ \\
\hline Cemento & 455.56 & 315.38 & 480.00 & 332.31 \\
\hline Grava & 741.12 & 793.60 & 870.54 & 937.15 \\
\hline Arena & 683.85 & 734.05 & 658.59 & 712.38 \\
\hline Agua & 205 & 205 & 216 & 216 \\
\hline Total & 2085.52 & 2048.04 & 2225.13 & 2197.83 \\
\hline
\end{tabular}

La cantidad de CBC adicionada fue de $10 \%$ respecto de la masa del CPC. Las mezclas se identificarán como: mezcla con $10 \%$ CBC y $100 \%$ GNT (CCR), mezcla con $10 \%$ CBC y $100 \%$ GNT (CCT), la mezcla con $100 \%$ GCR y fluidificante (TRF) y la mezcla con $100 \%$ GNT (TT).

\section{PROCEDIMIENTO}

\subsection{Elaboración de los especímenes.}

Se elaboraron especímenes prismáticos de $5 \mathrm{~cm}$ x $10 \mathrm{~cm}$ x $20 \mathrm{~cm}$. Fueron embebidas barras de acero de $3 / 8$ de pulgada de diámetro y se colocaron como electrodos de referencia barras de grafito en la cara 
de $5 \mathrm{~cm}$ x $10 \mathrm{~cm}$. Se realizó el curado por inmersión hasta lograr la pasivación del metal. Comprobado el estado pasivo se realizó la inmersión de las probetas en solución de $\mathrm{NaCl}$ al 3.5\% en masa.

\subsection{Pruebas electroquímicas}

La comprobación del estado pasivo y activo de las varillas se realizó con la prueba de potencial de corrosión $\left(\mathrm{E}_{\text {corr }}\right)$, utilizando como electrodos de referencia la barra de grafito y el electrodo de cobre/sulfato de cobre $\left(\mathrm{Cu} / \mathrm{CuSO}_{4}\right)$. Al realizar la inmersión en la solución de $\mathrm{NaCl}$ se realizaron mediciones de potenciales y Resistencia a la Polarización lineal ( $\mathrm{Rpl}$ ) en ciclos de humectación y secado, que consistieron en 7 días en inmersión y 21días fuera de la solución.

Para el cálculo de la velocidad de corrosión se utilizó la técnica de Rpl donde las pendientes con valores grandes corresponden a resistencias a la polarización lineal más altas y en general mejores condiciones protectoras del metal (Tórres Gómez \& et. al., 2010). Una vez obtenido el valor de Rpl, se procede a calcular la densidad de corriente de corrosión (i $\mathrm{i}_{\text {corr }}$ ) mediante la ecuación de Stern y Geary (1)-(2) (ASTM-G59, 2003).

$$
\begin{aligned}
& i_{\text {corr }}=10^{6} \frac{\mathrm{B}}{\mathrm{Rp}} \\
& B=\frac{\mathrm{b}_{\mathrm{a}} \mathrm{b}_{\mathrm{c}}}{\left[2.303\left(\mathrm{~b}_{\mathrm{a}}+\mathrm{b}_{\mathrm{c}}\right)\right]}
\end{aligned}
$$

Donde $i_{\text {corr }}$ es la densidad de corriente en $\mu \mathrm{A} / \mathrm{cm}^{2}$; B es el factor de Stern-Geary que depende de las pendientes de Tafel: anódica $\left(b_{a}\right)$ y catódica $\left(b_{c}\right)$, en $V ; R_{p}$ es la resistencia a la polarización en ohm$\mathrm{cm}^{2}$ (ASTM-G5, 2004).

\section{RESULTADOS}

\subsection{Potenciales de corrosión}

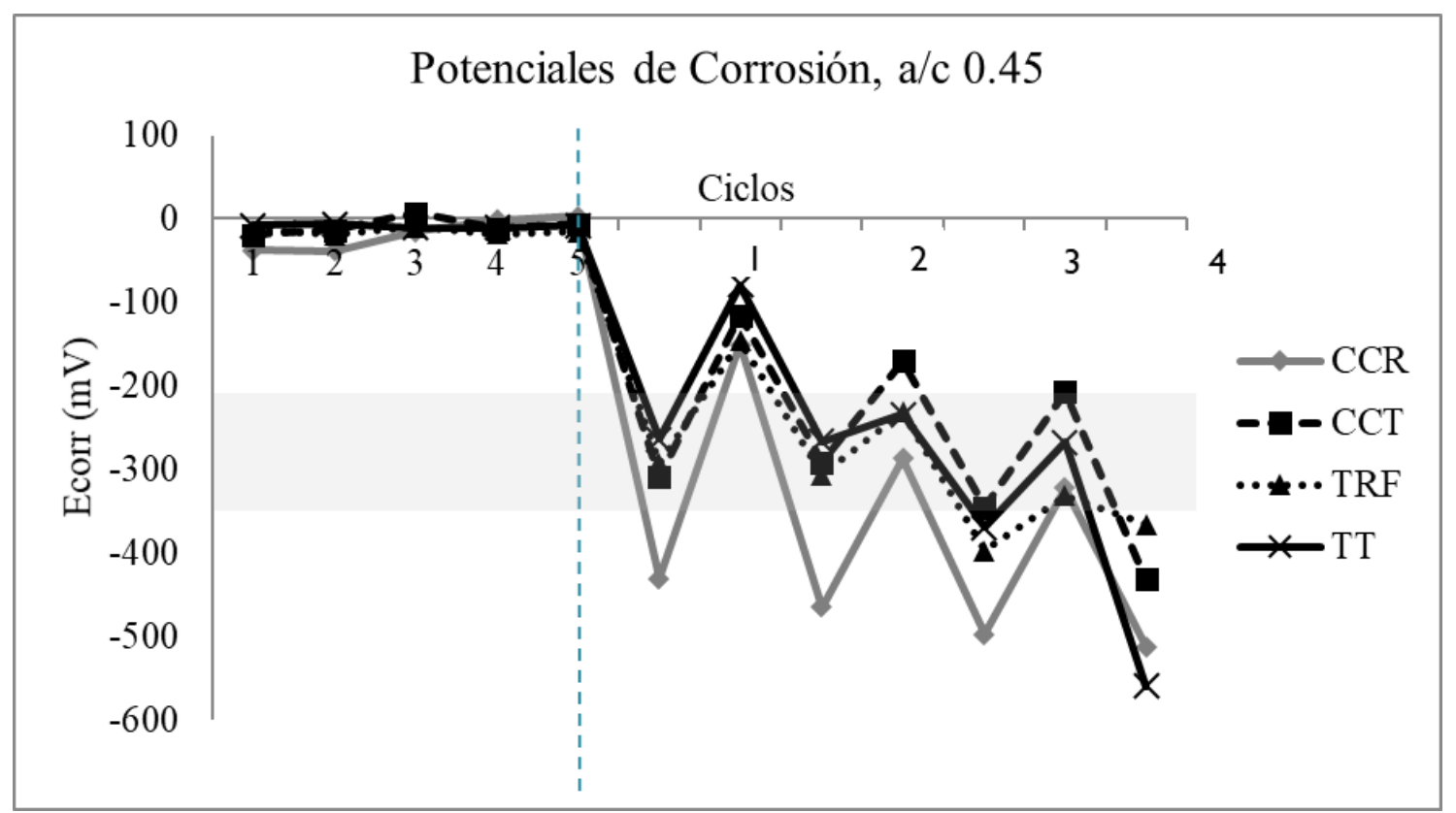

Figura 3 Potenciales de corrosión del acero embebido en las probetas de CCR, CCT, TRF y TT con relación a/c de 0.45 . Estado activo y pasivo, solución de $\mathrm{NaCl}$ al 3.5\%. 
En las Figuras 3 y 4 se muestran los resultados de los potenciales de corrosión. Se observa que las barras inmersas en los concretos elaborados con GNT presentan potenciales más positivos que los elaborados con GCR. Los valores más negativos se obtuvieron de las probetas elaboradas con la relación a/c de 0.65 , lo cual infiere mayor porosidad en la mezcla. Para a/c de 0.45 , en los tres primeros ciclos los valores de potenciales indican que existe incertidumbre sobre el fenómeno de corrosión en el acero, incluso estando más positivo que $-200 \mathrm{mV}$ en el primer ciclo, indicando probabilidad de $10 \%$ de ocurrencia del fenómeno de corrosión (NMX-C-495, 2015). La combinación más desfavorable para a/c de 0.45 es la de GCR y la CBC.

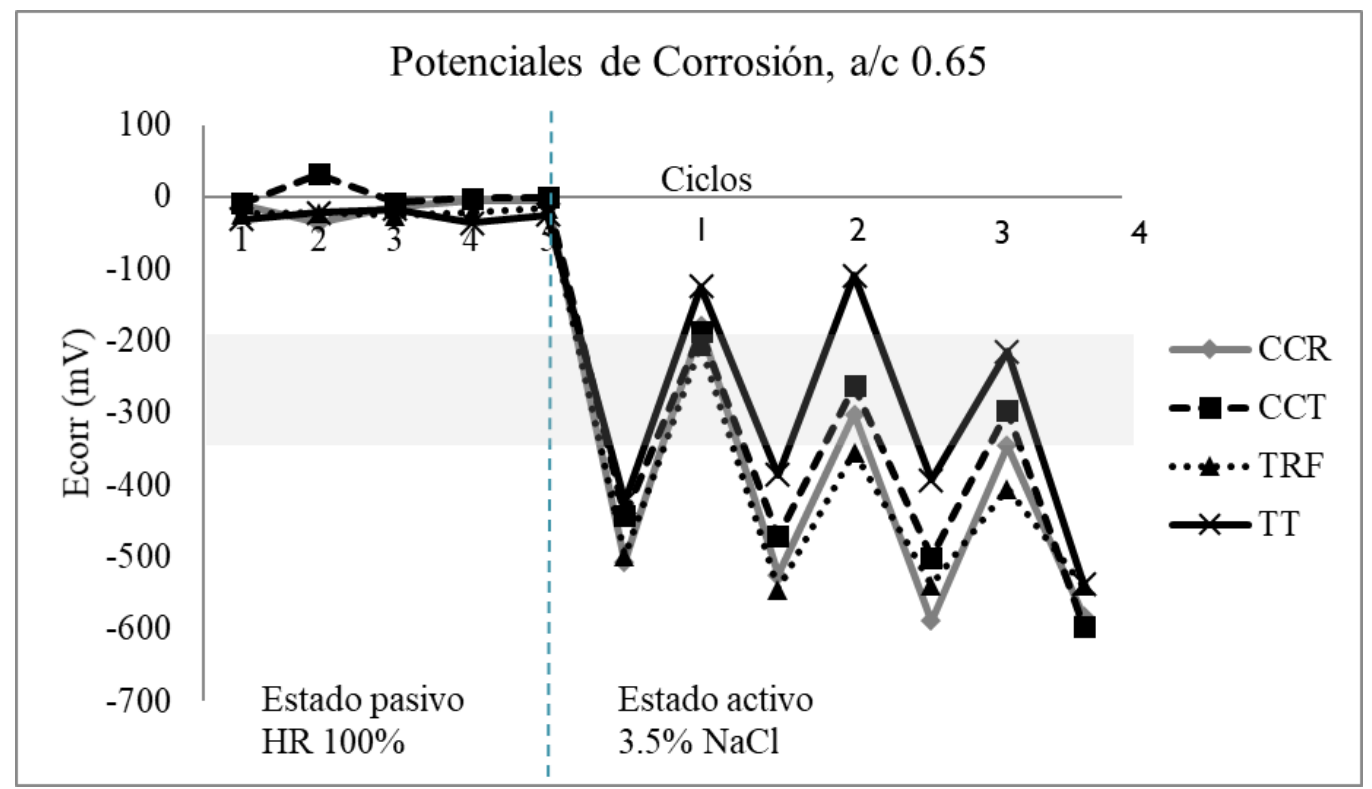

Figura 4 Potenciales de corrosión del acero embebido en las probetas de CCR, CCT, TRF y TT con relación a/c de 0.65. Estado activo y pasivo, solución de $\mathrm{NaCl}$ al 3.5\%.

\subsection{Velocidad de corrosión}

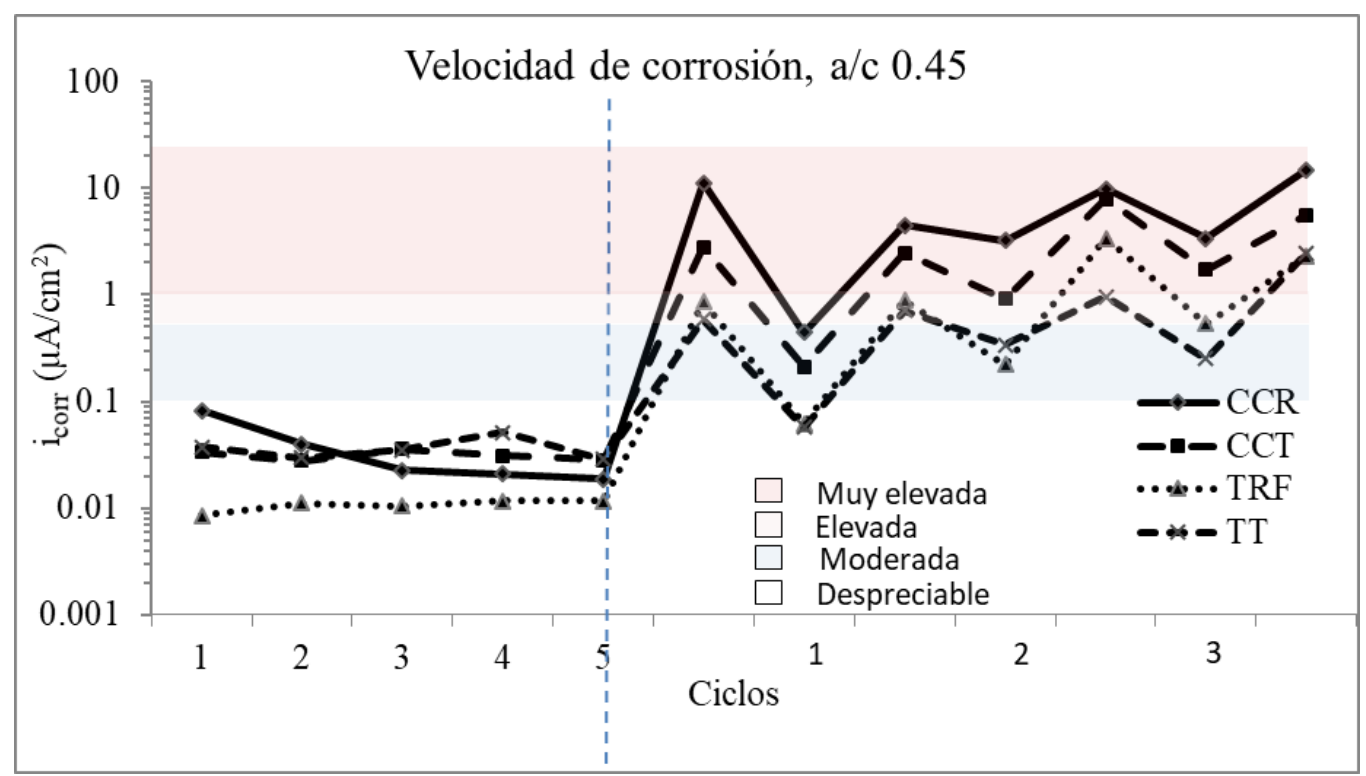

Figura 5 Velocidad de corrosión del acero embebido en las probetas de CCR, CCT, TRF y TT con relación a/c de 0.45 . Estado activo y pasivo, solución de $\mathrm{NaCl}$ al 3.5\%. 
En las Figuras 5 y 6 se observa que en el estado pasivo la mezcla que presenta una velocidad de corrosión menor, aun estando en un nivel despreciable, es la Testigo con GCR. Esto puede atribuirse a un incremento en la alcalinidad de la mezcla aportada por los álcalis del concreto reciclado.

En las probetas embebidas en las mezclas con relación a/c de 0.45 y adición de CBC se incrementa la velocidad de corrosión a un nivel muy elevado, mientras que los testigos con GNT y GCR oscilan entre moderada y elevada. Lo cual implica que la CBC no inhibe el fenómeno corrosivo.

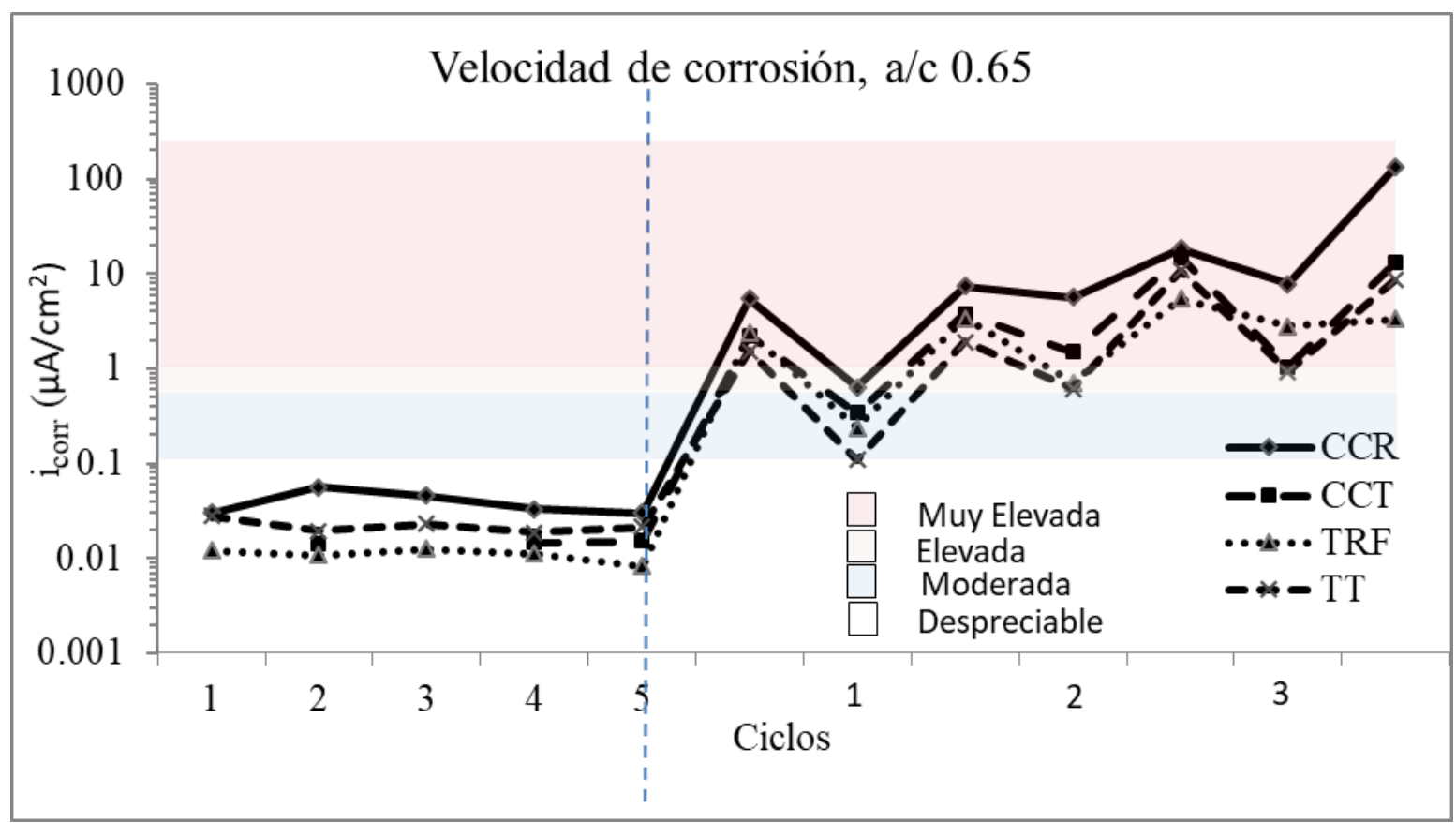

Figura 6 Velocidad de corrosión del acero embebido en las probetas de CCR, CCT, TRF y TT con relación a/c de 0.65. Estado activo y pasivo, solución de $\mathrm{NaCl}$ al 3.5\%.

En la figura 6 se observa como a partir del segundo ciclo las velocidades de corrosión se encuentran en un criterio de elevada a muy elevada, por lo que se comprueba que aun teniendo adiciones con reacción puzolánica y materiales alternativos como concreto reciclado, es imperativo el considerar una relación a/c para mejorar la durabilidad de las estructuras.

\section{CONCLUSIONES}

Las cenizas o residuos generados en procesos agroindustriales pueden integrarse como materiales de construcción alternativos. Como se sabe, la efectividad en las propiedades mecánicas ha sido analizada ampliamente en otros trabajos, sin embargo, al realizar experimentación incluyendo concreto reciclado se pueden hacer análisis para observar el fenómeno de corrosión en acero embebido en este tipo de compósito. La velocidad de corrosión del acero en los elementos con material reciclado y ceniza son altas comparadas con los elaborados con material natural triturado sin adición en medio salino, por lo que su aplicación es permitida en estructuras no expuestas a los agentes agresivos. En los años que están por transcurrir se cumplirá el tiempo de vida útil de muchas de las estructuras de concreto hidráulico construidas en el siglo pasado, por tal motivo tiene alta relevancia la investigación de las aplicaciones del concreto producto de demolición, para evitar que este tipo de materiales terminen en tiraderos a cielo abierto con aplicaciones nulas. 


\section{AGRADECIMIENTOS}

Se agradece al Laboratorio de Materiales de la Facultad de Ingeniería civil de la Universidad Michoacana por el apoyo en la realización de este trabajo, y por el apoyo económico al CONACYT.

\section{REFERENCIAS}

ASTM International. (2005). ASTM C150-05 Standard Specification for Portland Cement. Retrieved from https://doi.org/10.1520/C0150-05.

ASTM International. (2003). ASTM C618-03 Standard Specification for Coal Fly Ash and Raw or Calcined Natural Pozzolan for Use in Concrete. Retrieved from https://doi.org/10.1520/C0618-03.

ASTM International. (2004). ASTM G5-94(2004) Standard Reference Test Method for Making Potentiostatic and Potentiodynamic Anodic Polarization Measurements. Retrieved from https://doi.org/10.1520/G0005-94R04.

ASTM International. (1997). ASTM G59-97(2003) Standard Test Method for Conducting Potentiodynamic Polarization Resistance Measurements. Retrieved from https://doi.org/10.1520/G0059-97R03.

DURAR, R. (1998). Manual de Inspección. Evaluación y Diagnóstico de Corrosión en Estructuras de Concreto Armado. Río de Janeiro: CYTED Program.

Escalante, J., \& Navarro Gómez, A. (2011). Caracterización de morteros de cemento portland substituido por metacaolín de baja pureza.

Jagadesh, P., \& et. al. (2018). Evaluation of Mechanical Properties of Sugar Cane Bagasse Ash Concrete. Construction and Building Materials, 608-617.

Kosmatka, S. H., Kerkhoff, B., Panarese, W. C., \& y Tanesi, J. (2004). Diseño y Control de Mezclas de Concreto. Illinois, EE.UU: Portlan Cement Assosiation.

ONNCCE S. C. (2015). NMX-C-495. Industria de la Construcción-Durabilidad de Estructuras de Concreto Reforzado-Medición de Potenciales de Corrosión del Acero de Refuerzo sin Revestir, embebido en Concreto.

Pancorbo, F. J. (2013). Corrosión, degradación y envejecimiento de los materiales empleados en la edificación. México: Alfaomega.

Ruchansky, A. (2013). Posibilidades del uso de la ceniza de bagazo de caña como adición mineral al cemento Portland.

Tórres Gómez, R., Aperador, W., Vera , E., Mejía de Gutierrez, R., \& Ortiz, C. (2010). Study of Steel Corrosion Embedded In Aas. Dyna (164), 52-59.

Valdez Tamez, P. L., Das Roy, T. K., \& Rivera Villareal, R. (2004). Evaluación de la velocidad de Hidratación en sistemas puzolanas naturales-portlandita. En Ciencia UANL (Vol. VII, págs. 190-195). 